\title{
Polyakov-loop suppression of colored states in a quark-meson-diquark plasma
}

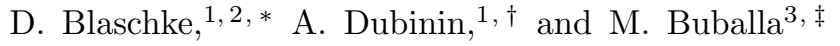 \\ ${ }^{1}$ Instytut Fizyki Teoretycznej, Uniwersytet Wroctawski, 50-204 Wroctaw, Poland \\ ${ }^{2}$ Bogoliubov Laboratory for Theoretical Physics, JINR Dubna, 141980 Dubna, Russia \\ ${ }^{3}$ Institute for Nuclear Physics, Technical University of Darmstadt, \\ Schlossgartenstr. 2, 64289 Darmstadt, Germany
}

\begin{abstract}
A quark-meson-diquark plasma is considered within the PNJL model for dynamical chiral symmetry breaking and restoration in quark matter. Based on a generalized Beth-Uhlenbeck approach to mesons and diquarks we present the thermodynamics of this system including the Mott dissociation of mesons and diquarks at finite temperature. A striking result is the suppression of the diquark abundance below the chiral restoration temperature by the coupling to the Polyakov loop, because of their color degree of freedom. This is understood in close analogy to the suppression of quark distributions by the same mechanism. Mesons as color singlets are unaffected by the Polyakov-loop suppression. At temperatures above the chiral restoration mesons and diquarks are both suppressed due to the Mott effect, whereby the positive resonance contribution to the pressure is largely compensated by the negative scattering contribution in accordance with the Levinson theorem.
\end{abstract}

PACS numbers: 12.39.Ki, 11.30.Rd, 12.38.Mh, 25.75.Nq

\section{INTRODUCTION}

For a quantum field theoretic description of hadronic correlations in quark matter that shares the property of approximate chiral symmetry with the QCD Lagrangian, the Nambu-Jona-Lasinio (NJL) model has been widely used. This model is particularly suitable to address the appearance of pions in quark matter as a consequence of dynamical chiral symmetry breaking in accordance with the Goldstone theorem. The absence of confinement is one of the shortcomings of the NJL model (see, e.g., [14 for early reviews and [5, 6, for more recent ones with emphasis on the high density aspects).

For a solution of this problem it has been proposed to take into account the suppression of colored quark states by their coupling to the Polyakov loop. For the Polyakovloop extended NJL (PNJL) model [7-10] in the mean field approximation a quark distribution function arises which in the limit of the confining phase (where for the traced Polyakov loop holds $\Phi \rightarrow 0$ ) is strongly suppressed relative to the ordinary Fermi function for quarks obtained in the deconfined phase where $\Phi \rightarrow 1$. Indeed this has been demonstrated in Refs. 8, 10. The model has been extended to also include mesonic correlations, see 12 17. Note that the coupling to the Polyakov-loop also suppresses thermodynamic instabilities in nonlocal NJL models [18, 19].

In the present work we want to investigate how the coupling of quarks to the Polyakov-loop will suppress the distribution of diquark states which arise from strong pairing correlations in quark matter. To this end we shall consider here the scalar diquark channel as a color an-

\footnotetext{
*Electronic address: blaschke@ift.uni.wroc.pl

${ }^{\dagger}$ Electronic address: aleksandr.dubinin@ift.uni.wroc.pl

‡Electronic address: michael.buballa@physik.tu-darmstadt.de
}

titriplet state and describe it in the framework of a generalized Beth-Uhlenbeck approach. Such a treatment was developed for describing excitonic correlations in semiconductor plasmas [20] and two-nucleon correlations in nuclear matter [21] before being adapted to the case of mesons in quark matter [22] and extended recently to the general case of two-quark correlations in quark matter [23.

This approach allows for a microscopic description of the occurrence of bound states in the equation of state of a nonideal plasma and their dissociation at high phase space densities due to the Mott effect. We develop this approach further by the coupling to the Polyakov loop analoguous to Ref. [24, see also [25, 26. Here we will demonstrate how the Polyakov-loop coupling leads to a strong suppression of the colored diquark states in the confining phase in straight analogy to the case of the color triplet quark states. As a striking elucidation of this effect we consider the superstrong coupling case for which the diquark becomes degenerate in mass to the pion so that the only difference between pion and diquark contributions to the thermodynamics are their numbers of degrees of freedom and the fact that pions are color neutral while the diquarks form a color antitriplet.

The present work can be considered an important step towards a description of baryons in quark matter. Any microscopic model which aims at this goal has to introduce diquarks first as elements in a Faddeev-type description of baryons as three-quark states (see, e.g., 27-29] for early works and [30, 31] for recent studies at finite temperature and chemical potential). Such a description has to explain why in the thermodynamics of quark matter with meson-, diquark-, and baryon correlations only mesons and baryons remain as observable degrees of freedom in the confined phase. With the present work we provide for the example of the PNJL model the still missing element, the suppression of diquark states in the confining phase. 


\section{MESONS AND DIQUARKS IN PNJL QUARK MATTER}

We base the approach on the PNJL model Lagrangian including diquark interaction channels besides the standard chirally symmetric scalar-pseudoscalar meson interaction for the isospin symmetric case $\left(\mu_{u}=\mu_{d}=\mu\right.$ and $\left.m_{u}=m_{d}=m_{0}\right)$

$$
\begin{aligned}
\mathscr{L}= & \bar{q}\left[\mathrm{i} \not \partial-m_{0}+\gamma_{0}\left(\mu-\mathrm{i} A_{4}\right)\right] q \\
& +\mathscr{L}_{\text {int }}-\mathscr{U}(\Phi, \bar{\Phi} ; T) \\
\mathscr{L}_{\text {int }}= & G_{\mathrm{S}}\left[(\bar{q} q)^{2}+\left(\bar{q} \mathrm{i} \gamma_{5} \tau q\right)^{2}\right] \\
& +G_{\mathrm{D}} \sum_{A=2,5,7}\left(\bar{q} \mathrm{i} \gamma_{5} \tau_{2} \lambda_{A} q^{c}\right)\left(\bar{q}^{c} \mathrm{i} \gamma_{5} \tau_{2} \lambda_{A} q\right) .
\end{aligned}
$$

Here, $q^{c}=C \bar{q}^{T}$ with $C=i \gamma^{2} \gamma^{0}$ denote the charge conjugate quark fields, $\lambda_{A}, A=2,5,7$, the antisymmetric Gell-Mann matrices in color space and $\tau_{i}, i=1,2,3$ the Pauli matrices in flavor space. $G_{S}$ and $G_{D}$ are dimensionful coupling constants. The Polyakov-loop potential $\mathscr{U}(\Phi, \bar{\Phi} ; T)$ is taken in the polynomial form [10, with the parameters taken from that reference. The homogeneous gluon background field in the Polyakov gauge is a diagonal matrix in color space $A_{4}=\lambda_{3} \phi_{3}+\sqrt{3} \lambda_{8} \phi_{8}=$ $\operatorname{diag}\left(\phi_{3}+\phi_{8},-\phi_{3}+\phi_{8},-2 \phi_{8}\right)$. The Polyakov loop field $\Phi$ is defined via the color trace over the gauge-invariant average of the Polyakov line $L(\vec{x})$ [10], which for homogeneous fields becomes rather simple

$$
\begin{aligned}
\Phi & =\frac{1}{N_{c}} \operatorname{Tr}_{c}\left[\exp \left(\mathrm{i} \beta A_{4}\right)\right] \\
& =\frac{1}{N_{c}}\left[\mathrm{e}^{\mathrm{i} \beta\left(\phi_{3}+\phi_{8}\right)}+\mathrm{e}^{-\mathrm{i} \beta\left(\phi_{3}-\phi_{8}\right)}+\mathrm{e}^{-2 \mathrm{i} \beta \phi_{8}}\right] .
\end{aligned}
$$

Its complex conjugate is denoted by $\bar{\Phi}$. Starting from the Lagrangian, we perform the usual bosonization by means of Hubbard-Stratonovich transformations, thus integrating out the quark degrees of freedom to obtain a path integral representation of the partition function (and thus the thermodynamical potential $\Omega$ ) in terms of composite fields, mesons $(M=\sigma, \vec{\pi})$ and (anti-)diquarks $\left(D, \bar{D}=\Delta_{A}, \Delta_{A}^{*}, A=2,5,7\right)$, which in Gaussian approximation can be evaluated in a closed form 23 .

$$
\mathscr{Z}_{\mathrm{Gau} B}=\mathscr{Z}_{\mathrm{MF}} \Pi_{X=M, D, \bar{D}} \mathscr{Z}_{X} .
$$

For the thermodynamic potential $\Omega=-(T / V) \ln \mathscr{Z}$ we obtain accordingly

$$
\Omega_{\mathrm{Gau} \beta}=\mathscr{U}(\Phi, \bar{\Phi} ; T)+\frac{\sigma_{\mathrm{MF}}^{2}}{4 G_{\mathrm{S}}}+\Omega_{\mathrm{Q}}+\Omega_{\mathrm{M}}+\Omega_{\mathrm{D}}+\Omega_{\overline{\mathrm{D}}}
$$

with

$$
\Omega_{\mathrm{Q}}=-\frac{1}{2} \frac{T}{V} \operatorname{Tr} \ln \left[\beta S_{\mathrm{Q}}^{-1}\right],
$$

containing the inverse quark propagator in the mean field approximation with the Nambu-Gorkov matrix representation

$$
S_{\mathrm{Q}}^{-1}=\left(\begin{array}{cc}
\left(\mathrm{i} z_{n}+\hat{\mu}\right) \gamma_{0}-\boldsymbol{\gamma} \cdot \mathbf{p}-m & \Delta_{\mathrm{MF}} \mathrm{i} \gamma_{5} \tau_{2} \lambda_{2} \\
\Delta_{\mathrm{MF}}^{*} \mathrm{i} \gamma_{5} \tau_{2} \lambda_{2} & \left(\mathrm{i} z_{n}-\hat{\mu}\right) \gamma_{0}-\gamma \cdot \mathbf{p}-m
\end{array}\right) .
$$

Here, $z_{n}=(2 n+1) \pi T$ are the fermionic Matsubara frequencies and we have introduced the combinations

$$
\begin{aligned}
m & =m_{0}+\sigma_{\mathrm{MF}}, \\
\hat{\mu} & =\mu-\mathrm{i} A_{4} \\
& =\operatorname{diag}\left(\mu-\mathrm{i} \phi_{3}-\mathrm{i} \phi_{8}, \mu+\mathrm{i} \phi_{3}-\mathrm{i} \phi_{8}, \mu+2 \mathrm{i} \phi_{8}\right) \\
& =\operatorname{diag}\left(\mu_{r}, \mu_{g}, \mu_{b}\right) .
\end{aligned}
$$

In the present work, we consider both cases, NJL and PNJL, but we will restrict ourselves to the normal phase without color superconductivity $\left(\Delta_{M F}=0\right)$.

Note that due to the presence of the diquark fields and the background gauge field the color trace is not trivial. In [23], we have neglected the gluon background field (NJL model: $A_{4}=0$ ) and performed an expansion w.r.t. the fluctuations of the composite fields (mesons and diquarks) around their (homogenous) mean field values up to Gaussian order where the path integral for the partition function can be evaluated in a closed form.

The functional trace $\operatorname{Tr}$ is defined as a sum over 4momenta times the trace tr over the internal degrees of freedom. In the infinite volume limit this becomes

$$
\operatorname{Tr}=\sum_{p_{n}} \operatorname{tr} \rightarrow V \sum_{z_{n}} \int \frac{d^{3} p}{(2 \pi)^{3}} \operatorname{tr}
$$

where $z_{n}$ denote the Matsubara frequencies, which are fermionic for quarks and baryons, and bosonic for mesons and diquarks. The trace in Dirac and flavor spaces in (5) is readily performed and after Matsubara summation we arrive for the quark thermodynamical potential at [8, 10] 


$$
\begin{aligned}
\Omega_{\mathrm{Q}}= & -2 N_{c} N_{f} \int^{\Lambda} \frac{d^{3} p}{(2 \pi)^{3}} E_{p}-2 N_{f} T \int \frac{d^{3} p}{(2 \pi)^{3}}\left\{\operatorname{tr}_{c=r, g, b} \ln \left[1+\mathrm{e}^{-\left(E_{p}-\mu_{c}\right) / T}\right]+\operatorname{tr}_{c=r, g, b} \ln \left[1+\mathrm{e}^{-\left(E_{p}+\mu_{c}\right) / T}\right]\right\}, \\
= & -2 N_{c} N_{f} \int^{\Lambda} \frac{d^{3} p}{(2 \pi)^{3}} E_{p}-2 N_{f} T \int \frac{d^{3} p}{(2 \pi)^{3}}\left\{\ln \left[\left(1+Y \mathrm{e}^{-\mathrm{i} \beta\left(\phi_{3}+\phi_{8}\right)}\right)\left(1+Y \mathrm{e}^{\mathrm{i} \beta\left(\phi_{3}-\phi_{8}\right)}\right)\left(1+Y \mathrm{e}^{2 \mathrm{i} \beta \phi_{8}}\right)\right]\right. \\
& \left.+\ln \left[\left(1+\bar{Y} \mathrm{e}^{\mathrm{i} \beta\left(\phi_{3}+\phi_{8}\right)}\right)\left(1+\bar{Y} \mathrm{e}^{-\mathrm{i} \beta\left(\phi_{3}-\phi_{8}\right)}\right)\left(1+\bar{Y} \mathrm{e}^{-2 \mathrm{i} \beta \phi_{8}}\right)\right]\right\}, \\
= & -2 N_{c} N_{f} \int^{\Lambda} \frac{d^{3} p}{(2 \pi)^{3}} E_{p}-2 N_{f} T \int \frac{d^{3} p}{(2 \pi)^{3}}\left\{\ln \left[1+3 \bar{\Phi} Y+3 \Phi Y^{2}+Y^{3}\right]+\ln \left[1+3 \Phi \bar{Y}+3 \bar{\Phi}^{2}+\bar{Y}^{3}\right]\right\},
\end{aligned}
$$

where we have introduced the abbreviations $Y=$ $\mathrm{e}^{-\left(E_{p}-\mu\right) / T}$ and $\bar{Y}=\mathrm{e}^{-\left(E_{p}+\mu\right) / T}$. Removal of the zeropoint energy term ("no sea" approximation) and integration by parts gives the thermodynamic potential in the form

$$
\Omega_{\mathrm{Q}}=-\frac{2 N_{c} N_{f}}{3} \int \frac{d p}{2 \pi^{2}} \frac{p^{4}}{E_{p}}\left[f_{\Phi}^{+}\left(E_{p}\right)+f_{\Phi}^{-}\left(E_{p}\right)\right],
$$

with the generalized Fermi distribution functions (cf. Ref. [12])

$$
\begin{aligned}
& f_{\Phi}^{+}\left(E_{p}\right)=\frac{(\bar{\Phi}+2 \Phi Y) Y+Y^{3}}{1+3(\bar{\Phi}+\Phi Y) Y+Y^{3}} \\
& f_{\Phi}^{-}\left(E_{p}\right)=\frac{(\Phi+2 \bar{\Phi} \bar{Y}) \bar{Y}+\bar{Y}^{3}}{1+3(\Phi+\bar{\Phi} \bar{Y}) \bar{Y}+\bar{Y}^{3}} .
\end{aligned}
$$

The limiting cases of the confined phase $(\Phi=\bar{\Phi}=0)$ and the deconfined phase $(\Phi=\bar{\Phi}=1)$ these distributions are the Fermi functions

$$
\begin{aligned}
\left.f_{\Phi}^{ \pm}(\omega)\right|_{\Phi=0} & =\frac{1}{\exp [3(\omega \mp \mu) / T]+1}, \\
\left.f_{\Phi}^{ \pm}(\omega)\right|_{\Phi=1} & =\frac{1}{\exp [(\omega \mp \mu) / T]+1} .
\end{aligned}
$$

In the confinement case, Fermi distribution functions with rescaled temperature arise $\left(T \rightarrow T / N_{c}\right)$, so that for a given temperature $T$ exponentially fewer quarks get excited than in the ordinary Fermi gas case for $\Phi \rightarrow 1$. The thermodynamic potential of the meson- and diquark channels in Gaussian approximation is

$$
\Omega_{\mathrm{X}}=\frac{1}{2} \frac{T}{V} \operatorname{Tr} \ln \left[\beta^{2} S_{\mathrm{X}}^{-1}\right], \mathrm{X}=\mathrm{M}, \mathrm{D}, \overline{\mathrm{D}} .
$$

where the inverse meson- and diquark propagators take the generic form

$$
S_{\mathrm{X}}^{-1}\left(\mathrm{i} z_{n}, \mathbf{q}\right)=\frac{1}{G_{\mathrm{X}}}-\Pi_{\mathrm{X}}\left(\mathrm{i} z_{n}, \mathbf{q}\right),
$$

with the polarization functions $\Pi_{\mathrm{X}}\left(\mathrm{i} z_{n}, \mathbf{q}\right)$ defined in the RPA approximation as one-loop integrals which involve combinations of quark-quark and quark-antiquark propagators with the corresponding vertex functions in the meson- and diquark channels, respectively. The required polarization loop integrals for mesons and diquarks are given in Ref. 23] for the NJL model. For the PNJL case, one has to replace in the final expressions the Fermi functions by the generalized Fermi distribution functions (12).

For the further evaluation of the thermodynamic potential (15) it is convenient to work in a polar representation of the complex propagator function which results from analytic continuation of (16) into the complex plane

$$
S_{\mathrm{X}}(\omega+i \eta, \mathbf{q})=\left|S_{\mathrm{X}}(\omega, \mathbf{q})\right| \exp \left[i \delta_{\mathrm{X}}(\omega, \mathbf{q})\right]
$$

and which defines the phase shift functions $\delta_{\mathrm{X}}(\omega, \mathbf{q})$. The medium dependence of these functions encodes important physical effects such as the merging of the discrete bound state part of the spectrum with the continuum of scattering states in the Mott transition. The details will be explained later in subsection IVB when discussing the numerical results of the present study. An expression for the second virial coefficient in the virial expansion of the quantum statistical partition function in terms of medium independent phase shifts has been given first by Uhlenbeck and Beth [32, who exploited the quantum mechanical models for hard sphere potential scattering. In this standard Beth-Uhlenbeck approach which is valid in the low-density limit one can also employ experimental data on two-particle scattering in free space, when they are available as, e.g., in the cases of Coulomb scattering [33, nucleon-nucleon scattering [34] or pion nucleon scattering [35].

The generalization of the Beth-Uhlenbeck EoS to higher densities has been developed in Refs. [20, 21]. Out of different aspects of this generalized Beth-Uhlenbeck approach it was in particular the modifications of the phase shifts due to a lowering of the continuum threshold for scattering states which entails the Mott dissociation of bound states that was interesting for the application to mesonic correlations in quark matter within the NJL model 22. What hindered a broad application of the generalized Beth-Uhlenbeck approach unifying mi- 
croscopic approach to the phenomenology of the hadronto-quark-matter phase transition was mainly the absence of quark confinement in the NJL model. With the advent of the PNJL models (see, e.g., 8 17]) the situation could be amended since colored quark excitations get suppressed by the coupling to the Polyakov-loop. In the present work we show how this Polyakov-loop suppression of colored states works for the diquark fields which are considered here together with their mesonic counterparts within the Polyakov-loop extension of the generalized Beth-Uhlenbeck approach to two-particle states in quark matter as derived in Ref. [23].

In the PNJL model the thermodynamic potential for a meson is not directly affected by the Polyakov loop and takes the same form as given in 23,

$$
\begin{aligned}
\Omega_{\mathrm{M}}= & d_{\mathrm{M}} T \int \frac{\mathrm{d}^{3} q}{(2 \pi)^{3}} \int_{0}^{\infty} \frac{\mathrm{d} \omega}{2 \pi}\left\{\ln \left(1-\mathrm{e}^{-\left(\omega-\mu_{\mathrm{M}}\right) / T}\right)\right. \\
& \left.+\ln \left(1-\mathrm{e}^{-\left(\omega+\mu_{\mathrm{M}}\right) / T}\right)\right\} \frac{d \delta_{\mathrm{M}}(\omega, \mathbf{q})}{d \omega}
\end{aligned}
$$

where $\mu_{\mathrm{M}}=\mu_{i}-\mu_{j}$ is the chemical potential of a meson $M$ composed of quark $i$ with chemical potential $\mu_{i}$ and antiquark $j$ with chemical potential $-\mu_{j}$. Since in the color singlet mesons the colors of quark and antiquark get neutralized, the meson chemical potential does not contain the gluon background fields $\phi_{3}$ and $\phi_{8}$. The me- son degeneracy factor is denoted by $d_{\mathrm{M}}$ and the vacuum contribution has been removed.

In such a formulation, the dissociation of the mesonic bound state in a hot, dense medium by the Mott effect is encoded in the behaviour of the in-medium phase shift. The analogous result for the diquark thermodynamic potential is derived in the next section for the PNJL model.

\section{COLOR TRACE FOR DIQUARK THERMODYNAMICAL POTENTIAL}

To obtain the diquark thermodynamics one starts from the bosonic thermodynamic potential (15) for the case $X=D$, taking the form 18 where the meson chemical potential $\mu_{M}$ has to be replaced by the diquark chemical potentials $\mu_{A}$ for the three diquark channels $D_{A}, A=$ $2,5,7$, which are diagonal matrices in color space

$$
\begin{aligned}
& \mu_{2}=\mu_{r}+\mu_{g}=2 \mu-2 \mathrm{i} \phi_{8} \\
& \mu_{5}=\mu_{r}+\mu_{b}=2 \mu-\mathrm{i}\left(\phi_{3}-\phi_{8}\right) \\
& \mu_{7}=\mu_{r}+\mu_{g}=2 \mu+\mathrm{i}\left(\phi_{3}+\phi_{8}\right)
\end{aligned}
$$

In the diquark thermodynamic potential which otherwise has the form similar to the pion one, the remaining color trace is evaluated in the following way

$$
\begin{aligned}
\Omega_{\mathrm{D}}= & \int \frac{d^{3} q}{(2 \pi)^{3}} \int \frac{d \omega}{2 \pi}\left\{3 \omega+T \operatorname{tr}_{A=2,5,7} \ln \left[1-\mathrm{e}^{-\left(\omega-\mu_{A}\right) / T}\right]+T \operatorname{tr}_{A=2,5,7} \ln \left[1-\mathrm{e}^{-\left(\omega+\mu_{A}\right) / T}\right]\right\} \frac{d \delta_{D}(\omega)}{d \omega}, \\
= & \int \frac{d^{3} q}{(2 \pi)^{3}} \int \frac{d \omega}{2 \pi}\left\{3 \omega+T \ln \left[\left(1-X \mathrm{e}^{-2 \mathrm{i} \beta \phi_{8}}\right)\left(1-X \mathrm{e}^{-\mathrm{i} \beta\left(\phi_{3}-\phi_{8}\right)}\right)\left(1-X \mathrm{e}^{\mathrm{i} \beta\left(\phi_{3}+\phi_{8}\right)}\right)\right]\right. \\
& \left.+T \ln \left[\left(1-\bar{X} \mathrm{e}^{2 \mathrm{i} \beta \phi_{8}}\right)\left(1-\bar{X} \mathrm{e}^{\mathrm{i} \beta\left(\phi_{3}-\phi_{8}\right)}\right)\left(1-\bar{X} \mathrm{e}^{-\mathrm{i} \beta\left(\phi_{3}+\phi_{8}\right)}\right)\right]\right\} \frac{d \delta_{D}(\omega)}{d \omega}, \\
= & \int \frac{d^{3} q}{(2 \pi)^{3}} \int \frac{d \omega}{2 \pi}\left\{3 \omega+T \ln \left[1-3 \Phi X+3 \bar{\Phi} X^{2}-X^{3}\right]+T \ln \left[1-3 \bar{\Phi} \bar{X}+3 \Phi \bar{X}^{2}-\bar{X}^{3}\right]\right\} \frac{d \delta_{D}(\omega)}{d \omega},
\end{aligned}
$$

where we have introduced the abbreviations $X=$ $\mathrm{e}^{-(\omega-2 \mu) / T}, \bar{X}=\mathrm{e}^{-(\omega+2 \mu) / T}$ and dropped the explicit notation of the three-momentum $\mathbf{q}$ as an argument of the phase shifts. This shorthand notation we shall use from now on. Removal of the zero-point energy term ("no sea" approximation) results in the diquark thermodynamic potential

$$
\begin{aligned}
\Omega_{\mathrm{D}}= & T \int \frac{\mathrm{d}^{3} q}{(2 \pi)^{3}} \int_{0}^{\infty} \frac{\mathrm{d} \omega}{2 \pi}\left\{\ln \left[1-3(\Phi-\bar{\Phi} X) X-X^{3}\right]\right. \\
& \left.+\ln \left[1-3(\bar{\Phi}-\Phi \bar{X}) \bar{X}-\bar{X}^{3}\right]\right\} \frac{d \delta_{\mathrm{D}}(\omega)}{d \omega} .
\end{aligned}
$$

While the mesons are color neutral the scalar diquarks are color antitriplet states and thus "see" the Polyakov loop. Note that in the confining phase it holds that $\Phi=$ $\bar{\Phi}=0$ which removes the contributions from one- and two- diquark states so that in this phase only the coherent sum of three diquarks with complementary colors can propagate. This effect is in full analogy to the case of colored quark excitations which are also suppressed by the Polyakov-loop factors in the confinement phase, see Eq. 10] 8, 10. 
After integration by parts 21] takes the form

$$
\Omega_{\mathrm{D}}=-3 \int \frac{d^{3} p}{(2 \pi)^{3}} \int \frac{d \omega}{2 \pi}\left[g_{\Phi}^{+}(\omega)+g_{\Phi}^{-}(\omega)\right] \delta_{D}(\omega),
$$

with the generalized Bose distribution functions

$$
\begin{aligned}
g_{\Phi}^{+}(\omega) & =\frac{(\Phi-2 \bar{\Phi} X) X+X^{3}}{1-3(\Phi-\bar{\Phi} X) X-X^{3}} \\
g_{\Phi}^{-}(\omega) & =\frac{(\bar{\Phi}-2 \Phi \bar{X}) \bar{X}+\bar{X}^{3}}{1-3(\bar{\Phi}-\Phi \bar{X}) \bar{X}-\bar{X}^{3}} .
\end{aligned}
$$

In the limiting cases of the confined phase $(\Phi=\bar{\Phi}=0)$ and the deconfined phase $(\Phi=\bar{\Phi}=1)$ these functions go over to the Bose functions

$$
\begin{aligned}
\left.g_{\Phi}^{ \pm}(\omega)\right|_{\Phi=0} & =\frac{1}{\exp [3(\omega \mp 2 \mu) / T]-1}, \\
\left.g_{\Phi}^{ \pm}(\omega)\right|_{\Phi=1} & =\frac{1}{\exp [(\omega \mp 2 \mu) / T]-1},
\end{aligned}
$$

which show that in the confined phase the thermal excitation of diquarks and antidiquarks is suppressed by a similar mechanism as the excitation of quarks and antiquarks.

This is the main new result of this work. In the following section we will obtain numerical results for it and discuss its consequences.

\section{RESULTS}

The parameters employed for the numerical studies are a bare quark mass $m_{0}=5.5 \mathrm{MeV}$, a three-momentum cutoff $\Lambda=639 \mathrm{MeV}$ and a scalar coupling constant $G_{\mathrm{S}} \Lambda^{2}=2.134$. We consider three values for the diquark coupling constant: (A) Fierz value: $G_{D} / G_{S}=3 / 4$, (B) strong coupling $G_{D} / G_{S}=1.0$ and (C) superstrong coupling: $G_{D} / G_{S}=1.5$. With these parameters one finds in vacuum a constituent quark mass of $319 \mathrm{MeV}$, a pion mass of $138 \mathrm{MeV}$ and pion decay constant $f_{\pi}=92.4$ $\mathrm{MeV}$. The vacuum mass of the $\sigma$-meson is $644 \mathrm{MeV}$, which is thus slightly unbound. The scalar diquark is unbound for the Fierz value of the coupling (case A) and bound for the strong and superstrong diquark couplings $\left(m_{D}^{(B)}=582 \mathrm{MeV}\right.$ for cases B and $m_{D}^{(C)}=m_{\pi}$ for case $\mathrm{C}$, resp.). In the present work, we restrict ourselves to applications at finite temperatures and vanishing chemical potential in the isospin-symmetric case of two-flavor quark matter.

\section{A. Mass spectrum in the NJL/PNJL model at finite temperature}

As a first step, the mean field gap equation for the quark mass is solved as a function of temperature for
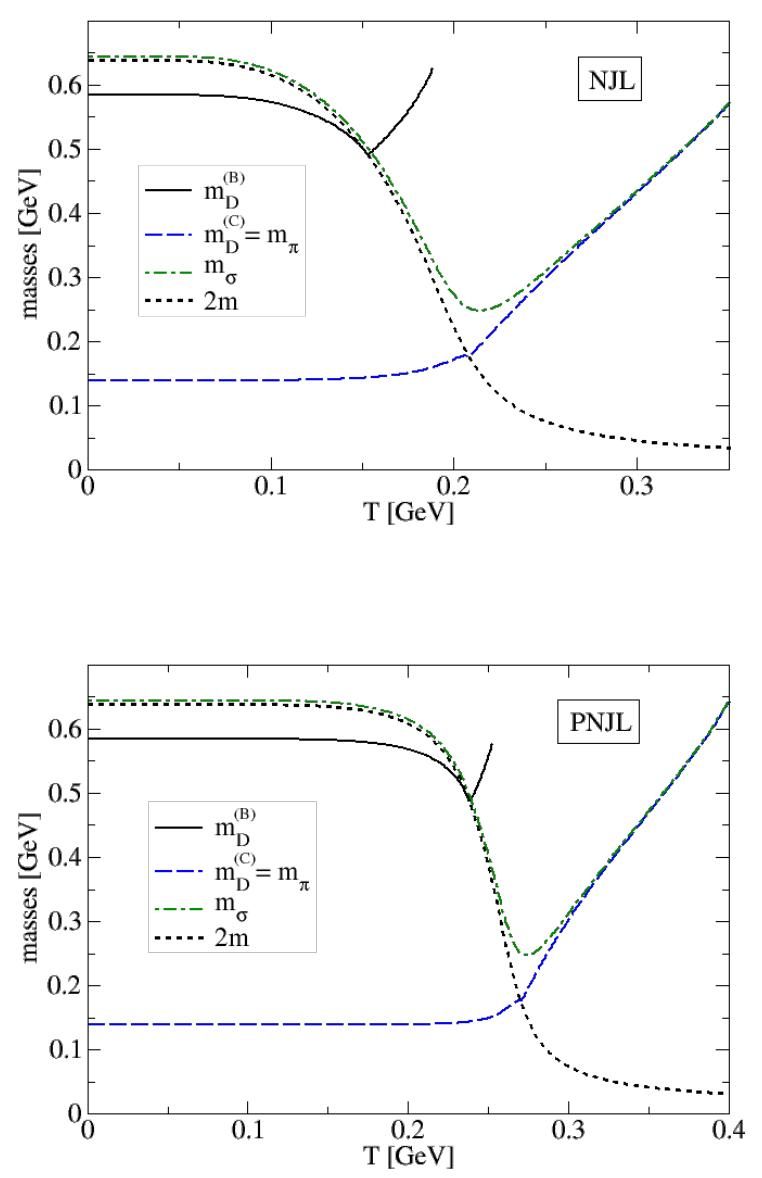

FIG. 1: Mass spectrum of pions, sigma mesons and diquarks as functions of the temperature $T$. The diquark mass $m_{D}^{(B)}$ $\left(m_{D}^{(C)}\right)$ corresponds to the case of strong (superstrong) diquark coupling. Also shown is the relevant threshold $2 m$ for mesons and diquarks. Upper panel: without Polyakov-loop; Lower Panel: with Polyakov-loop.

the NJL and PNJL models, respectively. These results serve as inputs for solving the Bethe-Salpeter equations for mesons and diquarks in the medium. The masses of quarks, pions, $\sigma$ mesons and diquarks are obtained as poles of their propagators and are shown in Fig. 1. We observe that the chiral symmetry restoration which is reflected in the dropping quark mass function, induces a Mott effect for the pion and the scalar diquark. For both states the kernel of their Bethe-Salpeter equation contains a Pauli blocking term since they are composed of two fermions. This Pauli blocking partly compensates the effect of the quark selfenergies (dropping masses) and leads to a stabilization of the bound state masses against medium effects. This results in the crossing of the bound state masses with the continuum threshold, leading to the dissociation of these bound states. 


\section{B. Phase shifts of mesons and diquarks in quark matter}

In the following, we will discuss the results for the diquark phase shifts (see also Refs. 23] ) and their consequences for the thermodynamics of quark-meson-diquark matter at finite temperature, with and without the coupling to the Polyakov-loop. The solution for the diquark phase shifts at finite temperature $(\mu=0)$ is shown in Fig. 2 as a function of the invariant mass variable $s$ for different temperatures. Here we made the simplifying assumption that, even in the medium, the phase shifts are Lorentz invariant and identify the function $\delta_{X}(s)$ with $\delta_{X}(\omega=\sqrt{s})$ calculated at rest $(\mathbf{q}=0)$ for given temperature and chemical potential of the medium. The bound state mass is located at the jump of the phase shift from 0 to $\pi$ and this jump corresponds to a delta-function in the Beth-Uhlenbeck formulas $(18)$ and $(21)$ for the correlations. In the case when the continuum of the scattering states can be neglected since it is separated by a sufficient energy gap from the bound state, we obtain the limiting case of thermodynamics of a statistical ensemble of onshell correlations.

\section{Beth-Uhlenbeck equation for mesons and diquarks in quark matter}

Now we want to study the thermodynamics of the meson and diquark correlations in a hot and dense medium encoded in the thermodynamic potentials (18) and 21), respectively.

In Fig. 3 we show the pressure as a function of the temperature for pions and diquarks within the NJL and the PNJL models, respectively. Let us note that within NJL-type models a general decomposition of the phase shifts into a resonant (R) and a continuum (c) part can be made

$$
\delta_{\mathrm{X}}(\omega)=\delta_{\mathrm{X}, \mathrm{R}}(\omega)+\delta_{\mathrm{X}, \mathrm{c}}(\omega),
$$

where both parts are uniquely defined by the propagator of the correlation (see Ref. 23]) and are important to establish accordance with the Levinson theorem in medium 24, 37.

$$
\int_{0}^{\infty} d \omega \frac{d \delta_{\mathrm{X}}(\omega)}{d \omega}=0 .
$$

The more conventional form of the Levinson theorem 36 introduces the energy level of the continuum threshold $\omega_{\mathrm{thr}}=\sqrt{q^{2}+m_{\mathrm{thr}}^{2}}$, where $m_{\mathrm{thr}}=2 m$ applies for the continuum of two-particle states (mesons, diquarks) composed of quarks with equal mass $m$

$$
\begin{aligned}
\int_{0}^{\omega_{\mathrm{thr}}} d \omega \frac{d \delta_{\mathrm{X}}(\omega)}{d \omega} & =-\int_{\omega_{\mathrm{thr}}}^{\infty} d \omega \frac{d \delta_{\mathrm{X}}(\omega)}{d \omega} \\
& =\delta_{\mathrm{X}}\left(\omega_{\mathrm{thr}}\right)-\delta_{\mathrm{X}}(\infty) .
\end{aligned}
$$
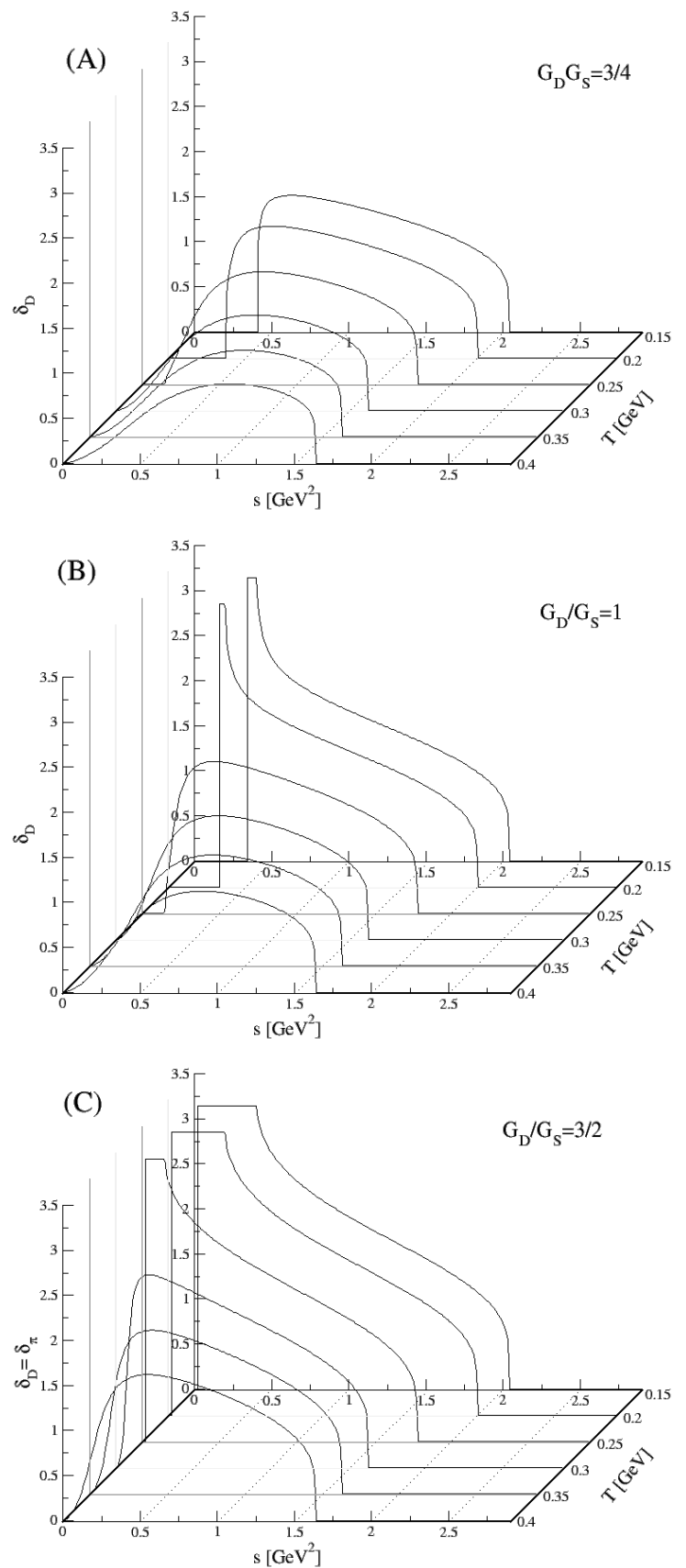

FIG. 2: Diquark phase shift $\delta_{D}$ as a function of squared center of mass energy $s$ at different temperatures from $T=150$ $\mathrm{MeV}$ to $400 \mathrm{MeV}$ for the three cases of diquark coupling: (A) Fierz value: $G_{D} / G_{S}=3 / 4$, (B) strong coupling $G_{D} / G_{S}=$ 1.0 and $(\mathrm{C})$ superstrong coupling: $G_{D} / G_{S}=1.5$. The jump of the phase shift from 0 to $\pi$ indicates the position of a bound state in the spectrum below the threshold of the continuum states which is situated where the phase shift starts decreasing towards zero. With increasing temperature the threshold moves to lower $s$-values and the phase shift jumps from $\pi$ to 0 when the Mott temperature is reached where the bound state gets dissociated. This behaviour is in accordance with the Levinson theorem. 
Since below the threshold can be only a discrete number $n_{\mathrm{B}, \mathrm{X}}$ of bound states in the channel $\mathrm{X}$, each contributing an amount of $\pi$ to the change in the phase shift at the bound state energies $\omega_{\mathrm{X}, \mathrm{i}}=\sqrt{q^{2}+m_{\mathrm{i}}^{2}}$ with $\mathrm{i}=1, \ldots, n_{\mathrm{B}, \mathrm{X}}$, it follows the Levinson theorem in the form

$$
\pi n_{\mathrm{B}, \mathrm{X}}=\delta_{\mathrm{X}}\left(\omega_{\mathrm{thr}}\right)-\delta_{\mathrm{X}}(\infty)
$$

which applies also in the case of a hot and dense medium.

In particular, when due to the chiral restoration the dropping mass of the quarks entails a lowering of the continuum threshold $\omega_{\text {thr }}$ which triggers the dissolution of the bound states into the continuum (the Mott effect), then $n_{\mathrm{B}, \mathrm{X}}=0$. In that case, it holds that $\delta_{\mathrm{X}}\left(\omega_{\text {thr }}\right)=$ $\delta_{\mathrm{X}}(\infty)$, which would be strongly violated if the phase shift (26) would be approximated by the resonance part only. This can be demonstrated, e.g., by employing a Breit-Wigner type ansatz for the phase shift

$$
\delta_{\mathrm{X}, \mathrm{R}}(\omega)=\frac{\pi}{2}+\arctan \left(\frac{\omega-\omega_{\mathrm{X}, \mathrm{R}}}{\Gamma_{\mathrm{X}}}\right),
$$

which would yield

$$
\begin{aligned}
\delta_{\mathrm{X}, \mathrm{R}}\left(\omega_{\mathrm{thr}}\right)-\delta_{\mathrm{X}, \mathrm{R}}(\infty) & =\arctan \left(\frac{\omega_{\mathrm{thr}}-\omega_{X, R}}{\Gamma_{X}}\right)-\frac{\pi}{2} \\
& \neq 0 .
\end{aligned}
$$

This violation of the Levinson theorem elucidates the importance of continuum background contribution to the phase shift 26).

In order to make the role of the Polyakov-loop suppression most explicit we have chosen in Fig. 3 the superstrong coupling case with the parametrization $G_{D}=$ $1.5 G_{S}$, for which the masses of pions and diquarks are degenerate. This is similar to the mass spectrum of two-color QCD [38 41]. It is this case for which the main result of this paper can be most clearly demonstrated, namely that the coupling to the Polyakov loop very effectively suppresses the partial pressure of the colored diquark states, thus making the thermodynamics of the PNJL model more "realistic" than that of the NJL model.

When we reduce the diquark coupling to the more realistic case (B), where the diquark mass is just below the continuum threshold $2 m$ and case (A) where it is even above the threshold, the diquark pressure is lowered further due to the increase in the diquark mass relative to case (C). In Fig. 4 we show the corresponding contributions to the diquark pressure as a function of the temperature for the NJL and the PNJL model, respectively.

We are now in a state to summarize the results for the thermodynamics of the quark-meson-diquark plasma within the PNJL model. In Fig. 5 we show the contributions from quarks, sigma mesons, pions and diquarks to the total pressure in hot quark matter for case (A), the Fierz value of the diquark coupling. We observe that
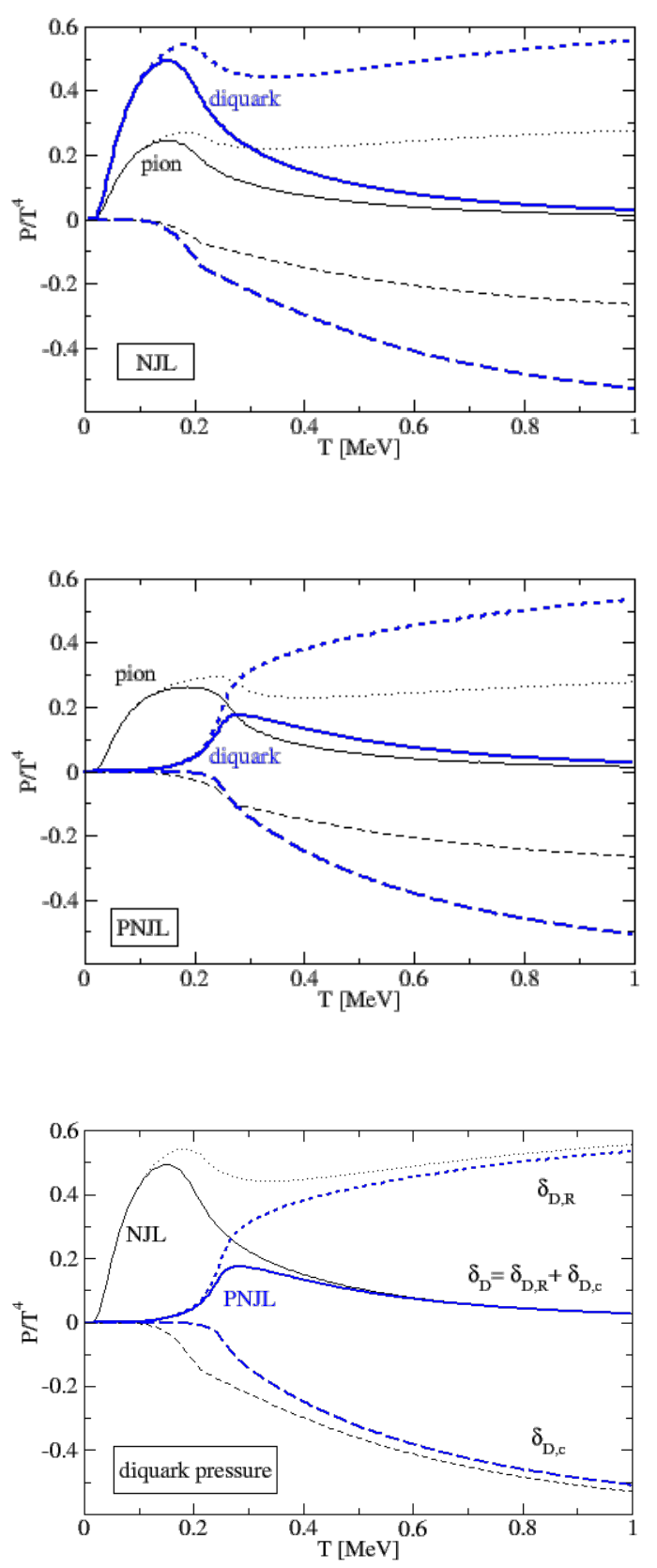

FIG. 3: Contributions from the resonant (positive) and scattering continuum (negative) parts to the total pressure (thick lines) for diquarks and pions in the NJL model (upper panel) and in the PNJL model (middle panel) as a function of temperature. In the lower panel a direct comparison is made of the diquark pressure with its resonant and continuum contributions for the NJL and the PNJL model, respectively, which demonstrates the strong suppression of the diquark pressure due to the Polyakov-loop coupling in the confining phase at low temperatures. In this figure we have chosen the superstrong coupling case (C) for which the masses of pions and diquarks are degenerate. 


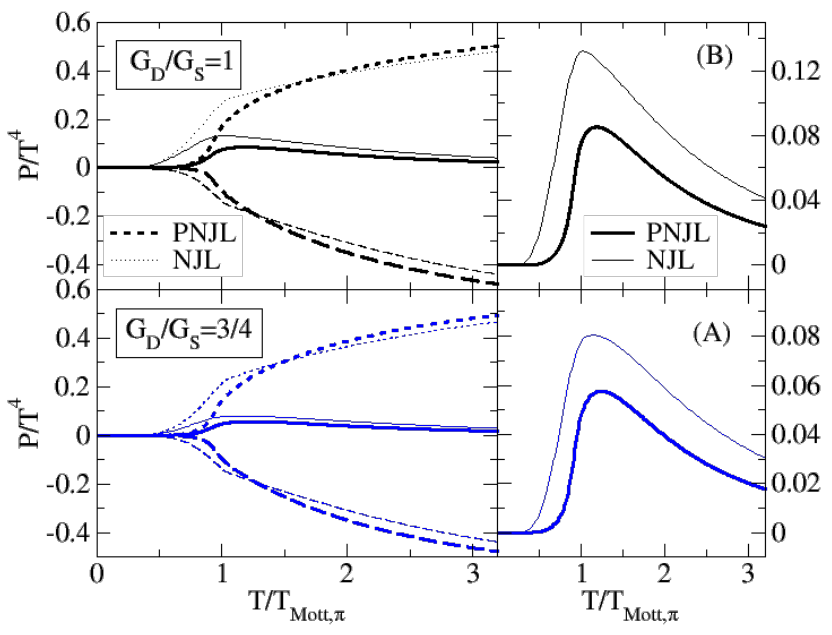

FIG. 4: Contributions to the diquark pressure in the NJL model (thin lines) compared to that of the PNJL model (thick lines) as a function of temperature: resonance (dotted lines), continuum (dashed lines) and total (solid lines) contributions for two cases of diquark coupling: strong (case (B), upper panels) and Fierz value (case (A), lower panels). The right panels demonstrate the suppression of the diquark pressure due to the Polyakov-loop coupling by comparing the NJL with the PNJL model results. The diquark pressure contains contributions from both, diquarks and antidiquarks.

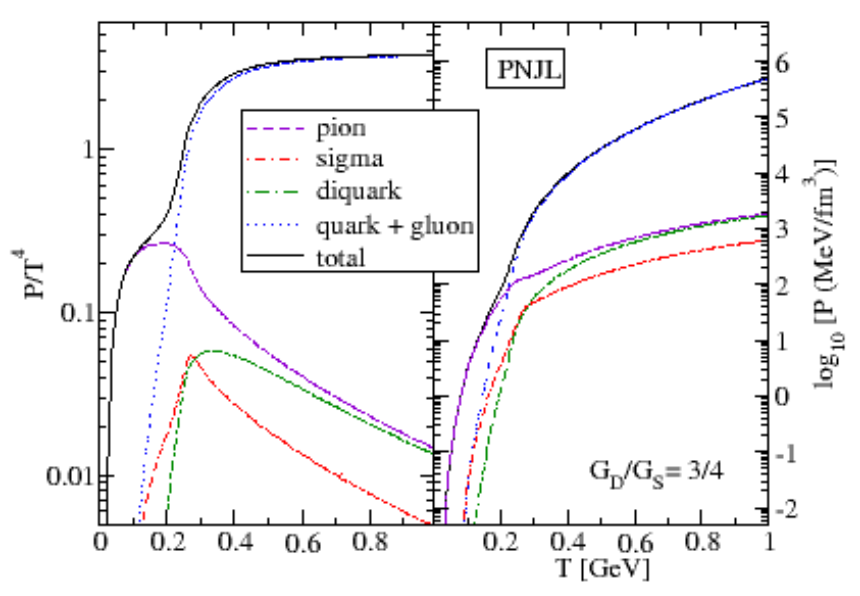

FIG. 5: Pressure of quark-gluon matter with meson and diquark correlations in the PNJL model as function of temperature (solid lines). Also shown are the partial pressures of quarks and gluons (dotted lines), diquarks (long-dash-dotted lines), sigma mesons (dash-dotted lines) and pions (dashed lines). The left panel shows the pressure contributions divided by $T^{4}$ (dimensionless) while on the right panel they are in units of $\mathrm{MeV} / \mathrm{fm}^{3}$.

for temperatures up to $T \sim 120 \mathrm{MeV}$ the total pressure is that of a pion gas while for temperatures exceeding
$T \sim 250 \mathrm{MeV}$ the thermodynamics is that of a quarkgluon plasma. The intermediate temperature region is that of the chiral restoration and deconfinement transition, where the system consists of a mixture of partons and pions, while heavier hadrons like the sigma meson as well as the diquarks are subdominant. In this transition region the composition of the thermodynamic system is governed by three suppression mechanisms for states: due to their mass, their spectral broadening (dissociation) and the Polyakov loop. While the mass suppresses states only at low temperatures, the spectral broadening acts for all composite states and suppresses them at high temperatures. The colored states (quarks and diquarks) are suppressed in the confined phase where the traced Polyakov loop is close to zero.

This result appears to capture the characteristic features of QCD thermodynamics at finite temperatures as it is simulated on the lattice [42, 43. However, before a quantitative comparison with lattice QCD can be attempted, more hadronic states need to be implemented as, e.g., in the recent phenomenological model [44 47]. Another important aspect is the consistent inclusion of correlation effects into the quasiparticle picture which is under way 48 .

\section{CONCLUSIONS}

A main result of this work is the derivation of the generalized distribution function for color $\mathrm{SU}(3)$ diquarks in a Polyakov-loop background field. In the limit of deconfinement, it goes over to the ordinary Bose distribution function while in the opposite case it is responsible for a strong suppression of the colored diquark state. This has been strikingly demonstrated by considering the superstrong coupling case $G_{D}=3 / 2 G_{S}$ for which the pion and diquark Bethe-Salpeter equations become degenerate and produce therefore the same solutions, masses and also phase shifts. In this case it is just the generalized Bose distribution function which leads to a strong suppression of the partial pressure of the colored diquark states relative to that of the color neutral pions. We have evaluated the composition (partial pressures) of quarkmeson-diquark matter in the two-flavor PNJL model as a function of the temperature at zero baryon density with the result that in the confinement phase the system becomes a pion gas where quarks and diquarks as colored degrees of freedom are suppressed by the Polyakovloop and sigma mesons by their mass. With increasing temperature the system undergoes the transition to the deconfined phase which coincides with chiral symmetry restoration. The meson and diquark contributions to the pressure are vanishing due to their dissolution in the Mott transition while their constituents become the dominant component, now forming a Fermi gas of colored states not suppressed by the Polyakov-loop since we are in the deconfined phase. 


\section{ACKNOWLEDGEMENT}

This work was supported by the Deutsche Forschungsgemeinschaft (DFG) under contract BU 2406/1-1 and by the Polish National Science Centre within the "Maestro" programme under contract UMO2011/02/A/ST2/00306. The work of D.B. was supported in part by the Hessian LOEWE initiative through HIC for FAIR. A.D. acknowledges a grant from the Institute for Theoretical Physics of the University of Wroclaw under contract No. 2470/M/IFT/14 and support by the Bogoliubov-Infeld programme for scientific collaboration between Polish Institutions and the JINR Dubna.
[1] M. K. Volkov, Annals Phys. 157, 282 (1984).

[2] U. Vogl and W. Weise, Prog. Part. Nucl. Phys. 27, 195 (1991).

[3] S. P. Klevansky, Rev. Mod. Phys. 64, 649 (1992).

[4] T. Hatsuda and T. Kunihiro, Phys. Rept. 247, 221 (1994).

[5] M. Buballa, Phys. Rept. 407, 205 (2005).

[6] K. Fukushima and C. Sasaki, Prog. Part. Nucl. Phys. 72, 99 (2013).

[7] P. N. Meisinger and M. C. Ogilvie, Phys. Lett. B 379, 163 (1996).

[8] K. Fukushima, Phys. Lett. B 591, 277 (2004).

[9] E. Megias, E. Ruiz Arriola and L. L. Salcedo, Phys. Rev. D 74, 065005 (2006).

[10] C. Ratti, M. A. Thaler and W. Weise, Phys. Rev. D 73, 014019 (2006).

[11] C. Sasaki, B. Friman and K. Redlich, Phys. Rev. D 75, 074013 (2007).

[12] H. Hansen, W. M. Alberico, A. Beraudo, A. Molinari, M. Nardi and C. Ratti, Phys. Rev. D 75, 065004 (2007).

[13] B. J. Schaefer, J. M. Pawlowski and J. Wambach, Phys. Rev. D 76, 074023 (2007).

[14] D. Blaschke, M. Buballa, A. E. Radzhabov and M. K. Volkov, Yad. Fiz. 71, 2012 (2008) [Phys. Atom. Nucl. 71, 1981 (2008)].

[15] S. Roessner, T. Hell, C. Ratti and W. Weise, Nucl. Phys. A 814, 118 (2008).

[16] A. E. Radzhabov, D. Blaschke, M. Buballa and M. K. Volkov, Phys. Rev. D 83, 116004 (2011).

[17] D. Horvatic, D. Blaschke, D. Klabucar and O. Kaczmarek, Phys. Rev. D 84, 016005 (2011).

[18] S. Benic, D. Blaschke and M. Buballa, Phys. Rev. D 86, 074002 (2012).

[19] S. Benic, D. Blaschke, G. A. Contrera and D. Horvatic, Phys. Rev. D 89, 016007 (2014).

[20] R. Zimmermann and H. Stolz, physica status solidi (b) 131, 151 (1985).

[21] M. Schmidt, G. Röpke and H. Schulz, Annals Phys. 202, 57 (1990).

[22] J. Hüfner, S. P. Klevansky, P. Zhuang and H. Voss, Annals Phys. 234, 225 (1994).

[23] D. Blaschke, M. Buballa, A. Dubinin, G. Röpke, D. Zablocki, Annals Phys. 348, 228 (2014).

[24] A. Wergieluk, D. Blaschke, Y. L. Kalinovsky and A. Friesen, Phys. Part. Nucl. Lett. 10, 660 (2013).
[25] K. Yamazaki and T. Matsui, Nucl. Phys. A 913, 19 (2013).

[26] K. Yamazaki and T. Matsui, Nucl. Phys. A 922, 237 (2014).

[27] R. T. Cahill, Austral. J. Phys. 42, 171 (1989).

[28] R. T. Cahill, J. Praschifka and C. Burden, Austral. J. Phys. 42, 161 (1989).

[29] H. Reinhardt, Phys. Lett. B 244, 316 (1990).

[30] J. -C. Wang, Q. Wang, D. H. Rischke, Phys. Lett. B704, 347 (2011).

[31] E. Blanquier, J. Phys. G 38, 105003 (2011).

[32] G. Uhlenbeck and E. Beth, Physica 3, 728 (1936); E. Beth and G. Uhlenbeck, Physica 4, 915 (1937).

[33] W. Ebeling, Ann. Physik 22, 33 (1968).

[34] C. J. Horowitz and A. Schwenk, Phys. Lett. B 638, 153 (2006).

[35] W. Weinhold, B. Friman and W. Norenberg, Phys. Lett. B 433, 236 (1998).

[36] R. Dashen, S. K. Ma and H. J. Bernstein, Phys. Rev. 187, 345 (1969).

[37] A. Dubinin, D. Blaschke and Y. L. Kalinovsky, Acta Phys. Polon. Supp. 7, 215 (2014).

[38] C. Ratti and W. Weise, Phys. Rev. D 70, 054013 (2004).

[39] N. Strodthoff, B. J. Schaefer and L. von Smekal, Phys. Rev. D 85, 074007 (2012).

[40] D. S. Zablocki, D. Blaschke and M. Buballa, Phys. Atom. Nucl. 75, 910 (2012).

[41] N. Strodthoff and L. von Smekal, Phys. Lett. B 731, 350 (2014).

[42] S. Borsanyi, G. Endrodi, Z. Fodor, A. Jakovac, S. D. Katz, S. Krieg, C. Ratti and K. K. Szabo, JHEP 1011, 077 (2010).

[43] A. Bazavov et al. [HotQCD Collaboration], Phys. Rev. D 90, 094503 (2014).

[44] L. Turko, D. Blaschke, D. Prorok and J. Berdermann, Acta Phys. Polon. Supp. 5, 485 (2012).

[45] L. Turko, D. Blaschke, D. Prorok and J. Berdermann, J. Phys. Conf. Ser. 455, 012056 (2013).

[46] L. Turko, D. Blaschke, D. Prorok and J. Berdermann, EPJ Web Conf. 71, 00134 (2014).

[47] D. Blaschke, A. Dubinin and L. Turko, Phys. Part. Nucl. 46, in press (2015); arXiv:1501.00485 [hep-ph]].

[48] D. Blaschke, PoS BaldinISHEPPXXII , 113 (2015); arXiv:1502.06279 [nucl-th]]. 